\title{
PUTNAM, QUINE Y LA "FACTICIDAD" DE LA SEMÁNTICA
}

Francisco A. Rodríguez-CONSUEGra

Derartamento de Lógica, Historia y Filosofía de la Ciencia

Facultad de Fllosofia, Universidad de Barcelona

La esencia general del argumento modelista de Putnam, manejado contra el "realismo metafísico", es que ningún criterio que fije los valores de verdad de los enunciados de un lenguaje es capaz de fijar la referencia de sus términos. De manera más precisa (1989), y partiendo de un lenguaje ya formalizado, la relación de referencia sería una función que asigne extensiones a las constantes individuales y a los símbolos de las funciones y los predicados, y que permita definir el conjunto de los enunciados verdaderos del lenguaje. Diríamos entonces que si existe tal cosa como una teoría ideal $I$, esa teoría no puede definir implícitamente su propia relación de referencia propuesta. En palabras de Putnam:

In fact, there are always many different reference relations that make $I$ true, if $I$ is a consistent theory wich postulates the existence of more than one object. Moreover, if $I$ contains non-observational terms (no matter how the line between the observational and the non-observational may be drawn), then there are reference relations that assign the correct extensions to the observation terms and wildly incorrect extensions to the non-observation terms and still make I come out true, as long as $I$ does not actually imply a false observation sentence.

Así, uno puede siempre hallar una relación de referencia que satisfaga las constricciones observacionales y las teóricas según las cuales tal teoría resulta verdadera, con lo que la relación de referencia lenguaje-realidad que necesita el realista, aunque existe, no es única, sino que da cabida a varios sistemas empíricamente equivalentes, todos los cuales disponen de una relación de correspondencia con la realidad que los hará verdaderos. Por último, ello hará que no podamos decir con sentido que una teoría ideal (que cumpla todas las constricciones) podría ser falsa, pues la verdad y la falsedad dependen sólo de la relación de referencia y de las constricciones exigidas.

A mí me parece que tal argumento es en esencia paralelo al que Quine nos ha dado para la inescrutabilidad de la referencia, lo cual no queda claro si uno 
lee lo que Putnam ha escrito sobre Quine en los último años, así que me ha parecido interesante localizar los pasajes relevantes de Putnam al respecto, así como llevar a cabo alguna discusión de su contenido con la vista puesta en las ideas de Quine, todo lo cual nos llevará a un punto en el que la divergencia es máxima: el problema de si hay o no hechos pertinentes en la semántica.

La postura explícita de Putnam respecto a las ideas centrales de Quine ha pasado por una curiosa evolución que podríamos resumir así: desde un rechazo absoluto, basado en una supuesta refutación, hasta una aceptación práctica de lo fundamental. Al mismo tiempo, Putnam insiste en que su punto de vista mantiene diferencias esenciales, sobre todo en lo referente a la facticidad de la semántica, donde Putnam está convencido de que Quine se equivoca. Por el contrario, a mí me parece que la postura de Quine sobre la semántica no es incoherente ni errónea, siempre que se admitan ciertos supuestos de partida, así que voy a insistir en que cuando Quine defiende la inexistencia de hechos pertinentes en la semántica no comete ningún error, por lo que puede acentuarse el paralelismo de su argumento de la inescrutabilidad de la referencia con el argumento modelista de Putnam.

En su "refutación del convencionalismo" Putnam (1962) acusó a Quine de cometer lo que llamó la "falacia convencionalista", consistente en una interpretación puramente hipotética de la indeterminación de la traducción según la cual Quine pasa de decir que ciertas constricciones no logran restringir el número de formas de escoger manuales de traducción, a decir que de hecho no puede haber constricciones adicionales que lo logren (sigo a Roth, 1986, p. 437). No merece la pena, sin embargo, comentar esta crítica pues se basa en un enfoque "práctico" de la traducción, mientras que Quine ha desarrollado con posterioridad sus ideas hasta la tesis fuerte de la relatividad ontológica (una versión algo más general de la inescrutabilidad de la referencia), que es precisamente la que sostengo que Putnam ha acabado aceptando de hecho, aparte de ciertas críticas menores y más bien verbales.

Sin embargo, sí es interesante plantear hasta qué punto puede hablarse de subdeterminación de la traducción, por asimilación a la subdeterminación de las teorías, puesto que Putnam habla en este artículo dando por sentado que la subdeterminación empírica de las teorías es, junto con la inescrutabilidad de la referencia, la base para la indeterminación de la traducción, con lo que podría quizá pensarse que en ambos casos se trata de una subdeterminación. $\mathrm{Y}$ es interesante porque si aceptamos el paralelismo en profundidad, podría quizá pensarse también que si en ambos casos hay subdeterminación, entonces también en ambos casos debe haber o no hechos pertinentes a la hora de comparar tanto teorías (esquemas conceptuales) empíricamente equivalentes como manuales de traducción indistinguibles (por respetar las disposiciones verbales), aunque incompatibles en numerosos puntos. 
Donde sí hay paralelismo desde el punto de vista de la subdeterminación es entre las teorías y la traducción radical, en el sentido de que en ambos casos cabe hablar de corrección (e incluso de verdad), pues ambos tienen que adaptarse a la evidencia disponible. Tanto cuando comprobamos la verdad de un enunciado (dentro de una teoría), como cuando lo hacemos con la traducción de un enunciado (dentro de un sistema de hipótesis analíticas), hemos de hacerlo con respecto a lo observable posible. Podemos pues hablar de subdeterminación en ambos casos, aunque no de indeterminación. En palabras de Quine: nuestras teorías y creencias están insuficientemente determinadas por la totalidad de la evidencia sensible posible, igual que la traducción radical de enunciados lo está por la totalidad de disposiciones al comportamiento verbal.

Eso significa, sin embargo, que en ambos casos hay hechos pertinentes, al menos en el sentido de que recurriendo a ellos deberíamos poder rechazar ciertas teorías o ciertas traducciones. Donde no los hay es a la hora de comparar entre dos traducciones que respeten por igual las disposiciones al comportamiento verbal, puesto que no hay nada que cambie la naturaleza a la hora de distinguir entre sistemas de hipótesis analíticas, dado que no podemos recurrir a un significado objetivo: la referencia es inescrutable. Podría entonces pensarse que lo mismo cabría decir al comparar dos teorías empíricamente equivalentes, pero no sería correcto, dado que en este caso sí hay un sustrato mediante el cual hablar con sentido de verdad: sólo que resulta referido a cada teoría, por lo que no podemos salirnos de dos teorías alternativas y afirmar que las dos son verdaderas.

Además, Quine ha insistido en que la indeterminación de la traducción se deriva más bien del holismo y el pragmatismo que de la subdeterminación de las teorías propiamente dicha. Puede argumentarse, por ejemplo, que la traducción es indeterminable sin más que decir que no hay sustrato de significado (o de creencia) al que recurrir para determinar "lo que realmente se quiere decir", o "lo que realmente se cree", excepto si se acepta que existe un museo mental en el que residen tales supuestos contenidos esenciales. Así, sólo podemos comunicarnos eficazmente a través del pragmatismo de las disposiciones verbales, inscrito en el contexto de un esquema conceptual que dota de sentido a cada parte componente. Por lo tanto, aunque a veces Quine hable como si la subdeterminación de las teorías llevase a la indeterminación de la traducción, en realidad no necesitamos recurrir a ella para hacerlo, con lo que el peligro de que asimilemos ambas a la subdeterminación, y por tanto a la existencia de hechos pertinentes, puede evitarse.

En su conversión a lo que ha llamado "realismo interno", basado en la primera versión del argumento modelista, Putnam (1978) se limita a escribir que el argumento (basado en el teorema de Löwenheim-Skolem) "tiene que ver con lo que Quine llama 'relatividad ontológica'" (p. 133), pero sin la debida exégesis, a excepción de ciertos vagos comentarios acerca de la posibilidad de 
interpretar unas teorías en otras de muchas formas diferentes y la consiguiente conclusión de la imposibilidad de hablar de una sola teoría correcta, en todo lo cual vemos, no sólo una relación lejana con Quine, sino un seguimiento de su línea general, a falta de que se señalen diferencias relevantes. Máxime cuando el artículo termina con una referencia adicional a Quine donde Putnam dice estar de acuerdo con él en que una supuesta verdad absoluta incorregible (que Putnam considera como idealización) hay que considerarla sólo como un límite inalcanzable, y relaciona todo ello con un cierto kantismo blando y desmitificado.

En Putnam 1980, que a veces se presenta como el lugar canónico del nuevo realismo interno, Putnam nos endosa una versión algo más desarrollada del argumento, de nuevo basado en el teorema de Löwenheim-Skolem, pero en cuanto a Quine, todo lo que se nos dice es que hay dos líneas en la filosofía de la ciencia actual, una la heredera de Ramsey, que finalmente aboga por una relatividad de los términos teóricos, y otra realista, cuya ala radical estaría ocupada precisamente por Quine (p. 434):

El ala pragmática radical, representada quizá por Quine, está dispuesta a abandonar la intuición de que $T_{I}$ [la teoría "ideal"] podría ser falsa "en realidad". Esta ala radical es "realista" en cuanto que está dispuesta a sostener que la ciencia actual, tomada más o menos al pie de la letra (esto es, sin reinterpretación filosófica) es al menos aproximadamente verdadera; y es "realista" en cuanto que considera la referencia como transteórica (una teoría con un enunciado de Ramsey verdadero puede ser falsa, porque la investigación posterior puede establecer que una teoría incompatible es mejor); pero no es realista metafisica.

Sin embargo, como veremos más abajo, aunque puede admitirse el primer matiz realista (al que sería necesario, sin embargo, añadir matices relativos sobre todo a los hechos "pertinentes"), en cambio no así el segundo: es sabido que para Quine la referencia es inescrutable en general, pero decir que es transteórica parece significar que se suscribe alguna oscura teoría metafísica de la correspondencia. No obstante es sorprendente que Putnam, a pesar de eximir a Quine del realismo metafísico, no relacione su propio realismo interno con la postura del viejo maestro.

En su Reason, Truth and History (1981) Putnam no es más preciso respecto a Quine, pero añade afirmaciones dignas de comentario. Lo más sorprendente es que presenta su nueva versión del argumento modelista (ahora sin ninguna referencia al teorema de Löwenheim-Skolem y por tanto válido ya para lógicas superiores) como una prolongación "radical" de los resultados de Quine respecto a la indeterminación (p. 44). Sin embargo el núcleo del argumento es el acostumbrado, es decir: "ningún criterio que únicamente fije los valores de verdad de oraciones completas puede fijar la referencia". La supuesta prolongación radical podría consistir, por tanto, en las palabras inmediatamente 
siguientes: "incluso si especifica los valores de verdad de las oraciones en cada mundo posible", con lo que la generalización se referiría a la lógica intensional. Lo de lo intensional podría también referirse a que al no depender ya más del teorema de Löwenheim-Skolem, válido sólo para lenguajes de primer orden, el argumento vale ya para otras lógicas (Putnam lo subraya en una nota de 1989). Sin embargo, Quine no ha hecho nunca depender su argumento del teorema de Löwenheim-Skolem, ni por tanto tampoco lo ha limitado a la lógica de primer orden, por más que para Quine, como es sabido, no hay más lógica que ésta. (Su utilización del teorema, como veremos más abajo, tuvo lugar principalmente para mostrar un posible problema de la reducción ontológica: el del peligro pitagorista.) Además, Putnam cita un pasaje de Quine (p. 52) que desarrolla una versión general de su argumento de la indeterminación de la referencia (que equivale a la relatividad ontológica) sin señalar ninguna diferencia relevante con su propia postura.

Afortunadamente hay una pista para establecer esa diferencia, y ésta tiene lugar cuando Putnam, después de presentar la tesis de Quine como "semejante" (p. 46), añade que para éste las condiciones de verdad para las oraciones "subdeterminan la referencia", con lo que da la impresión de que Quine está hablando de teorías empíricas, mientras que, como es sabido, Quine ha establecido una diferencia entre el orden de la traducción, donde no hay hechos pertinentes ni hay "verdad" (salvo en sentido especificado más arriba), y el orden de las teorías empíricas, donde sí los hay, aunque con los matices correspondientes, que veremos más abajo. Putnam añade:

uno podría salir de Palabra y objeto con la impresión de que todas las reinterpretaciones que no alteran el valor veritativo de una oración están al menos estrechamente relacionadas con la interpretación estándar (del mismo modo que las "partes de conejo" y la "conejidad" están relacionadas con conejos). El argumento detallado en el Apéndice e ilustrado en este capítulo demuestra que las condiciones de verdad para "Un gato está en una estera" ni siquiera excluyen la posibilidad de que "gato" se refiere a cerezas.

Sin embargo esto no constituiría diferencia alguna. Si Quine hablaba en 1960 de interpretaciones cercanas a la estándar es porque desarrolló su argumento en el orden de la traducción radical práctica, pero tanto en esa obra como en otras posteriores ha matizado y generalizado su argumento precisamente en el sentido remarcado por Putnam. No parece pues haber base convincente para hablar de "generalización de Quine". Pero veamos la postura de Quine con algo más de detalle.

El argumento general de la inescrutabilidad de la referencia se formuló en $\S 7$ de Palabra y objeto de manera completamente general: "es posible permutar la totalidad infinita de las sentencias del lenguaje de cualquier individuo dado, de tal modo que a) se mantenga invariante la totalidad de las disposiciones del 
individuo por lo que respecta al comportamiento real, y, sin embargo, b) la proyección no sea una mera correlación de sentencias con otras equivalentes en ningún sentido plausible de equivalencia, por laxo que sea". Por otra parte, Quine dice también que los enunciados categóricos dependen para su verdad de los objetos de los que son verdaderos los términos componentes, pero lo que sean tales objetos no está univocamente determinado por las significaciones estimulativas (p. 74), lo cual parece dar base de nuevo para conceder la máxima generalidad al argumento de la inescrutabilidad de la referencia. Es claro que con ello Quine no hace ninguna referencia específica a un lenguaje formalizado, como hace Putnam con su argumento, pero eso no ha de interpretarse como si el argumento no fuese válido para lenguajes formales, o con alguna otra restricción. Como es sabido por su polémica con Carnap, para Quine no es admisible una distinción última entre lenguajes naturales y artificiales, ya que sólo podemos construir un lenguaje artificial por extensión de los mecanismos que ya dominamos en nuestro lenguaje natural (por similitud con su rechazo de los lenguajes privados). Lo cual significa simplemente que sólo podemos entender un lenguaje artificial si proyectamos sobre él el lenguaje que es real para nosotros, y ése es de carácter social, etcétera, etcétera.

Quine ha insistido posteriormente con detalle en que su argumento es de una gran generalidad, sin aparentes limitaciones, por ejemplo en La relatividad ontológica (p. 69): dada una red de términos, predicados y términos auxiliares, que funciona como un esquema de referencia, o sistema de coordenadas, podemos situarlo todo, pero también podemos considerar denotaciones alternativas: "una gran e ingeniosa permutación de estas denotaciones, con los ajustes compensatorios en las interpretaciones de las particulas auxiliares, puede acomodar todas las disposiciones de habla". Así, no hay más referencia que la relativa a un sistema de coordenadas dado previamente, de la misma forma que no hay velocidad o posición absolutas. De nuevo: "Carece de sentido decir, dentro de una teoría, cuál de los varios posibles modelos de nuestra forma teórica es nuestro modelo real o pretendido. Todavía puede tener sentido decir, dentro de una teoría, que tenga ella muchos modelos. Podemos mostrar que para cada uno de los modelos, aunque inespecificable, existe inevitablemente otro que es una permutación o quizá una disminución del primero" (p. 75). Esto demuestra que Quine es máximamente general. Al mismo tiempo, su forma natural de argumentar conecta de manera inequívoca con los modelos no estándar, así como con el problema de la reducción (al hablar de disminución), temas a los que me referiré más abajo.

El tercer volumen de los Philosophical Papers de Putnam (1983) ofrece nuevas tomas de postura respecto a Quine, en las que salen ya a relucir diferencias más profundas. Para ser breve señalaré sólo dos. La primera tiene lugar cuando Putnam describe lo que llama "relatividad ontológica" mediante el consabido argumento de la falta de una interpretación privilegiada para los 
términos de nuestros lenguajes. Sin embargo ahora (1983, pp. xii-xiii) dice rechazar esa postura, mientras que, como hemos visto, en 1981 no sólo decía aceptarla, sino que incluso presentaba su postura como una generalización de ella. El argumento que justifica el rechazo es que bajo semejante relatividad la noción de objeto adquiere un carácter metafísico inaceptable, que Putnam profundiza acusando a Quine de mantener la idea de que existe una correspondencia entre el lenguaje y el mundo, por más que no una correspondencia determinada. Parece pues que Putnam no establece ninguna diferencia entre lo que se refiere al lenguaje y lo que se refiere a la ciencia empírica, diferencia que, como vengo señalando, es vital para entender a Quine. Como veremos más abajo, mientras cabe hablar de correspondencia y de verdad, esto sólo es posible para teorías científicas, pero no para la semántica, aunque correspondencia y verdad sean siempre relativas a una teoría determinada.

La segunda crítica de Putnam está también relacionada con el mismo problema. Putnam (1983, pp. 240 ss.) dice que la visión de Quine (1975) de que las teorías cientificas axiomatizadas deben capacitar para predecir (de forma económica) enunciados observacionales verdaderos desde el punto de vista de la teoría del significado como estímulo es inconsistente con su teoría de la verdad, pues según ésta todo enunciado científico debe ser verdadero o falso, y por Gödel sabemos que siempre habrá en tales teorías enunciados indecidibles, mientras Quine, que rechaza el realismo metafísico, diría que sin embargo tales enunciados deben tener también un valor de verdad aunque nosotros lo desconozcamos. Por otro lado, añade Putnam, la naturalización de la epistemología que defiende Quine le lleva a ver ésta como una rama de la psicología, con lo que no se ve claro cómo mantener lo que de normativo hay en cualquier noción como la de justificación, fiabilidad o verdad. Parece, por tanto, una vez más, que la incoherencia la ve Putnam justamente en el hecho de que mientras por un lado se defienda la verdad, por otro se haga dentro de un marco naturalista, de manera parecida, diría yo, a aquella en la que desde los "programas fuertes" (que exigen una explicación similar tanto para las creencias falsas como para las verdaderas) se defienden ambas nociones. Si ello fuera así, estaríamos ante otra versión de la dificultad señalada más arriba, que surgiría al conciliar el hecho de que mientras en la semántica no habría hechos pertinentes, sí los habría en la ciencia empírica.

En la última sección de su contribución al Schilpp de Quine (1986) Putnam dedicó algunos párrafos a criticar la negativa quineana a admitir hechos pertinentes, con lo que nos muestra de nuevo que el problema radica sobre todo en la admisión de la postura de Quine relativa a tales hechos. Putnam argumenta ahora sobre la base de que no podemos pasar de la afirmación de que todo cambio es un cambio fisico (que dice aceptar), a la de que todo hecho es un hecho físico (que dice rechazar), por lo que no debemos aceptar que no haya tales hechos pertinentes en el campo del significado o la refe- 
rencia. Así, prosigue Putnam, es antiintuitivo que no existan tales hechos con sólo afirmar que los enunciados referidos al significado tienen sólo un valor de verdad relativo a una teoría: “'Gorbeh means cat' may be true relative to our present mentalistic linguistic 'theory', but there is an empirically equivalent theory relative to which that statement is not true, and there is no "fact of the matter' as to which of these empirically equivalent theories is correct" (p. 425). Por último, Putnam añade que si el argumento fuese aceptable, sería también aplicable a la filosofia, con lo que la filosofía tomista, por ejemplo, sería también compatible con los hechos de la naturaleza (las "trayectorias de los cuerpos").

En su réplica Quine dedica algún espacio a cada una de esas críticas. En primer lugar, Quine aclara que sobre lo que no hay hechos pertinentes es sólo acerca de cuál de dos manuales completos de traducción compatibles con la evidencia disponible es el correcto (suponiendo que haya uno correcto, dado que son incompatibles). Pero eso no sucede en el caso de la determinación de ciertas traducciones concretas a la hora de construir un manual dado, puesto que en este caso sí hay evidencia relevante disponible: la relativa a la sinonimia que depende del estímulo que se suscita (sinonimia estimulativa): "there is a fact of the matter that 'Gavagai' is stimulous-synonymous with 'Rabbit'" (p. 429). Con ello parece que Putnam no distingue, o al menos no lo hace con la suficiente claridad para sus lectores, entre el caso en el que tratamos de discernir si una determinada traducción de un término se adecua a la evidencia observable disponible (para lo cual obviamente sí hay hechos pertinentes), con el otro caso, completamente diferente, para el cual se trata de discriminar entre dos manuales de traducción completos, que se adecuan igualmente bien con toda la evidencia observable disponible, por más que sean incompatibles en numerosos puntos, en el que no habría hechos pertinentes de ningún tipo. (Por incompatibilidad no se quiere aquí decir más que los diferentes manuales, una vez aplicados a la traducción, proporcionarían, como traducción de un enunciado de partida, enunciados que no serían equivalentes entre sí en ningún sentido del térnino.)

En cuanto a la identificación entre hechos pertinentes y estados microfísicos, Quine es igualmente claro: puesto que los hechos pertinentes de la naturaleza que tienen que ver con la corrección de una traducción son las disposiciones verbales, está claro que diferentes mauales de traducción incompatibles pueden coincidir con los mismos estados microfísicos. Por último, Quine admite francamente su cientificismo, pero lo conecta con su naturalismo, y con el hecho de que para él la filosofía no es más que una parte de nuestra imagen científica del mundo, por lo que se supone que nuestra filosofía ha de ser compatible con lo que nos diga la ciencia, $y$ aquí la física tiene sin duda un lugar insustituible. 
En todo caso podríamos añadir por nuestra parte que ése es el sentido en el que para Quine podemos pasar de la afirmación de que todo cambio es físico a la subsiguiente según la cual no hay más hechos que los hechos físicos. Con ello lo único que afirma es una voluntad, plenamente científica, de deshacerse metodológicamente de las entidades mitológicas aportadas por los mentalistas, como ocurre con los significados "privados", que olvidan, no ya que el lenguaje es social, sino incluso que toda observación lo es. Ése es el sentido de cientificismo que Quine ha defendido en otros lugares, donde queda claro de nuevo que, contra Putnam, puede decirse que sólo en lo físico hay hechos pertinentes, mientras que lo semántico no implica cambios con trascendencia física (respuesta a Alston, Hahn y Schilpp, 1986, pp. 74 s.):

In my view [...], the underdetermination of both disciplines is quite on a par methodologically. The asymmetry lies elsewhere than in methodology, as I shall try to explain. It may be helpfull to forget about theoretical physics; I can express my point in another way. We can agree, I hope, that one's command of language, one's understanding of language, one's disposition to respond, indeed one's very thoughts, cannot differ from one moment to another without some difference, however undetectable, in the states of one's physical organism. Call the doctrine materialism or psychophysical parallelism; either will do. Now the relation between the interchangeable but incompatible manuals of translation that I have postulated is that they accord with exactly the same states of human organisms, however minutely modulated, all the same hidden states of nerves. This is the sense in which I say there is no fact of the matter. I am talking not of criteria, but of nature.

Con ello queda eficazmente respondida la crítica de Putnam al cientificismo de Quine. Es cientificismo, sí, pero no le produce la menor incoherencia a la hora de comparar sus tesis semánticas con sus tesis sobre ciencia empírica. En todo caso, tal cientificismo no ha llevado a Quine a defender la ilusión de que sus doctrinas filosóficas puedan asimilarse a hipótesis empíricas, de lo que Putnam a veces le acusa. Una bonita manera de desmarcarse de tal asimilación ha consistido en su observación de que "no hay hechos pertinentes acerca de si hay o no hechos pertinentes" (Putnam, 1975, p. 177).

Por lo que sé, Putnam no ha contrarreplicado a tales argumentos de forma directa, pero en su 1989 se extiende por primera vez algo más sobre estos problemas. En primer lugar Putnam aborda por fin la similaridad de su argumento modelista con el de Quine, aunque lamentablemente se limita a repetir que hay "interesantes semejanzas" (p. 228), añadiendo que las diferencias radican sólo en que él no se ocupa de las "proxy functions" y además extiende el argumento a la lógica intensional (p. 221), todo lo cual lo previno, termina Putnam, de aceptar la versión de Quine, que llevaba directamente a negar la facticidad de la semántica. 
Pero las funciones sustitutivas son exactamente lo mismo que lo que Quine llama manuales de traducción, sólo que aplicadas al caso específico de la reducción ontológica, por lo que fueron introducidas por Quine con objeto de eliminar la posibilidad pitagorista implícita en la reducción trivial procedente del teorema de Löwenheim-Skolem. Según Quine, el problema se resuelve introduciendo la necesidad de funciones sustitutivas que conecten las entidades que han de reducirse con las entidades reductoras. Por tanto el argumento general no depende en absoluto de tales funciones, así que en este caso la supuesta diferencia no es relevante (véase mi 1992a). Posteriormente Quine ha mostrado más claramente la aplicación de las proxy functions al caso del lenguaje ordinario y la traducción. En Theories and Things (p. 29-30) Quine ha escrito que las funciones sustitutivas pueden también aplicarse, con independencia del tema de la reinterpretación reductiva, al mismo caso de la reinterpretación general en la que no hay propiamente reducción (pues no eliminamos nada), sino que nos limitamos a permutar los objetos sin cambiar en nada la estructura de partida ni tampoco su apoyo empírico, que es naturalmente el caso de la traducción alternativa. De ahí surge de nuevo la inescrutabilidad de la referencia, pero con el lenguaje de la traducción, en lugar del de las teorías o la reducción: determinar los objetos de los que alguien habla es sólo decir cómo proponemos traducir sus términos a los nuestros. En consecuencia la nueva aplicación de las funciones sustitutivas no sirve tampoco para diferenciar el argumento de Putnam del de Quine.

En cuanto a la supuesta generalización, ya decíamos más arriba que sería rechazada por Quine con sus conocidos argumentos contra lo intensional. De no ser así él mismo se habría encargado de llevarla a cabo. Para Quine no hay más lógica genuina que la lógica de primer orden, siendo la de segundo orden teoría de conjuntos disfrazada, y las lógicas intensionales rechazables. Así, aunque ha de admitirse que Quine no ha hecho nada por extender su argumento central a tales lógicas, lo cierto es que carecía del menor interés en hacerlo. Por consiguiente, es dudoso que eso constituya una diferencia significativa, o que reste potencia al argumento de Quine.

En segundo lugar, Putnam sitúa el problema principal precisamente en la diferencia que señalábamos más arriba: en el hecho de que Quine niegue la existencia de hechos objetivos relativos a la referencia y la sinonimia, mientras los admite para los enunciados observacionales (p. 221). Para colmo, Putnam insiste en el hecho de que ya muchos críticos han acusado a Quine de inconsecuencia al negar hechos pertinentes en lingüística y admitirlos en física (p. 224). Putnam cita el libro de Hookway sobre Quine, donde éste defiende al viejo maestro de semejante ataque aduciend̦o una diferencia entre epistemología (que regiría las traducciones reales, prácticas) y metafísica (válida en última instancia), sin embargo el propio Putnam insiste en que se trata de un error de Quine, a quien ahora acusa de confundir hecho con hecho físico, 
con lo que en la senda de Chomsky termina defendiendo el paralelismo entre semántica y fisica, que se confirma así como el núcleo de sus críticas a Quine.

Afortunadamente podemos aclarar la postura de Quine, y con ello tratar de cerrar la polémica, atendiendo a sus últimos escritos relevantes, que se concretan en Teorias y cosas (1981) y diversos "replies" en el trabajo de Schilpp (1986) dedicado a su obra, todo ello publicado, pues, con anterioridad al artículo de Putnam de 1989, en el que, sin embargo, no se encuentran las referencias relevantes. Parte de ese trabajo ha sido espléndidamente realizado ya por Gibson (1986), con la bendición explícita y entusiasta del propio Quine, asi que comenzaré por señalar brevemente el argumento principal, que le sirve a Gibson para contradecir a Rorty, Føllesdal, Chomsky y otros, aunque curiosamente no a Putnam, a pesar de que lo que critica en ellos es precisamente sus dificultades para admitir la distinción quineana entre semántica y física respecto a los hechos pertinentes.

La clave del asunto está en ver que, aunque la indeterminación de la referencia y la subdeterminación de las teorías son paralelas desde el punto de vista metodológico (epistemológico), que trata de cuestiones de evidencia, no lo son sin embargo desde el punto de vista ontológico, que se ocupa de lo que hay, es decir, de la verdad. Sin embargo la relación entre epistemología y ontología es sutil, pues, aunque diferentes, cada una de ellas está contenida de una forma distinta en la otra. En efecto, la epistemología está contenida en la ontologia, pero sólo en el sentido de que Quine es empirista, y por tanto su única evidencia admitida es la evidencia sensorial, por lo que su ontología queda determinada por el método científico, y no por ningún tipo de mera coherencia. Así, su naturalización de la epistemología presupone inevitablemente la ontología de la ciencia natural. En cambio, aunque también la ontología está contenida en la epistemología, lo está sólo en el sentido de que "es ella misma una proyección del mismo tipo de estimulaciones que se atribuyen al sujeto humano de su estudio", lo que equivale a desterrar todo a priori de nuestra ontología de la ciencia natural.

A partir de ahí el problema de la asimetría entre semántica y física se aclara. Así, aunque es cierto que traducciones alternativas son igualmente aceptables desde el punto de vista observacional, ello no reclama verdad alguna puesto que no hay hechos en la epistemología, ni pertinentes ni de ningún tipo. No hay pues nada extraño en que muchas traducciones sean igualmente aceptables observacionalmente y no haya nada más que escrutar. El panorama en ontología es muy diferente. Aunque dos teorías se hallen igualmente subdeterminadas por la evidencia observable, es decir, aunque sean equivalentes desde el punto de vista epistemológico, no tiene sentido decir de ellas que pueden ser igualmente verdaderas. $Y$ no lo tiene porque, aunque pueden estar igualmente garantizadas, nosotros no podemos hablar desde un punto de vista privilegiado; no podemos adoptar un nivel que trascienda ambas teorías, ya que la 
verdad es siempre inmanente a una teoria dada. Y en cuanto nos situamos dentro de una teoría dada entonces si hay hechos pertinentes. El misterio queda así desvanecido.

Pero pueden darse más argumentos, al tiempo que se profundiza en los problemas implícitos. Un argumento adicional sería el siguiente. Podemos asimilar el problema de la traducción al de la reducción. Con ello, la "verificación" de una traducción quedaría limitada a comprobar si cumple con los requisitos de comunicación necesarios. $Y$ esto significa, en el caso de la reducción, si las entidades a las que hemos reducido las originales cumplen con las funciones que se esperan de las entidades a reducir. Por ejemplo, lo que ocurre en el caso de las diversas reducciones conjuntistas de los números. En este caso particular, que creo es ampliable a otros, tenemos que las diversas "traducciones" conjuntistas cumplen por igual las funciones básicas que se espera cumplan los números. Por tanto, no hay "facts of the matter" que nos permitan escoger entre dos formas de reducción concretas, dado que todas ellas cumplen con lo esperado. Un posible fallo de este enfoque sería que no está del todo claro que aquí se cumplan los requisitos de "sinonimia", en el sentido de suponer que las diversas lecturas conjuntistas permitieran la comunicación, como ocurría con Ernie y Johnnie en el célebre artículo de Benacerraf. Pero Quine reconoció que con las diversas reducciones no había que esperar sinonimia. Bastaría por tanto decir que los aspectos no coincidentes quedarían "fuera" del conjunto de exigencias o requisitos esperables.

Otro argumento surge de forma natural con sólo reflexionar sobre la negativa de Quine a admitir el mito del significado: no hay verdad al comparar dos traducciones porque primero tendría que haberla en la determinación del significado, cosa que no existe. Más en detalle: no cabe decir que una traducción es mejor que otra (admitiendo que preservan por igual la conducta o la comunicación) si concedemos que traducir bien presupone determinar el significado correctamente. Ahora bien, no hay significado (esencia) que determinar, si admitimos la tesis de la inescrutabilidad de la refereacia (dejando de lado ahora si esto depende a su vez de la subdeterminación de las teorías). Así, no hay nada de que protestar si podemos establecer de hecho una comunicación globalmente exitosa partiendo de manuales de traducción diferentes. En consecuencia, no tenemos nada a que recurrir para hacer la comparación entre ellos, pues concedemos que todas las consecuencias verificables coinciden. Resumiendo: como no hay significado, tampoco hay forma de comparar entre dos traducciones, si las interpretamos como formas de determinar el significado correcto.

Una posible objeción para este enfoque sería que bajo tales supuestos la expresión "determinar el significado" tiene un sentido preciso: comprobar que las consecuencias verificables coinciden, con lo que aunque no podemos determinar el significado, sí podemos hablar con sentido de la "igualdad de 
significados", o sinonimia. Entraríamos así en el campo de la definición por abstracción y podríamos en principio definir la igualdad de significados con base en la posibilidad de la traducción misma: dos enunciados significan lo mismo si y sólo si uno traduce al otro, lo cual se verifica con base en la preservación de las conductas correspondientes. Pero, como ya Putnam señaló (1975, p. 179), el problema es que lo que demuestra Quine es que hay muchos isomorfismos diferentes de un lenguaje a otro que preservan la conducta observable, por lo que la noción misma de igualdad de significado resulta relativa al isomorfismo particular que escojamos, y puede por tanto cambiar.

Así, el argumento anterior, extraído de la reducción, conserva su validez: seguimos sin poder hablar del "significado" (en sí) de un enunciado, pues éste se apoya subrepticiamente en (presupone) un isomorfismo dado. Por volver al ejemplo de las diversas reducciones conjuntistas de los números, tampoco podríamos decir que la reducción conjuntista de von Neumann determina el mismo significado para los números que la de Zermelo, pues cada una depende de un isomorfismo diferente. Aquí, como en la traducción, no cabe hablar de "verdad", aunque cabe hablar de reducción-traducción correcta. El paralelismo es sin embargo más profundo, pues así como la verdad es relativa a una teoría o esquema conceptual, la sinonimia entre lenguajes es relativa a un sistema particular de hipótesis analíticas sobre el que elaboramos cada manual de traducción.

Roth (1986, p. 440) ha dado un argumento semejante, aunque más simple, para insistir en que no hay hechos en la semántica, sobre la base de que aceptarlos sería aceptar que antes de llevar a cabo una traducción, estamos en disposición de determinar el significado de un término y dejarlo fijado, para después compararlo con el propuesto por la traducción que nos propongan. Pero tal determinación previa es ya imposible, por lo que no cabe la comparación posterior. Pero quizá podría aducirse contra Roth que, como Quine ha escrito en algún lugar, decir que no haya hechos pertinentes en la semántica no es decir que no los haya a la hora de preparar "un" manual dado de traducción, sino sólo que no los hay a la hora de comparar entre dos de ellos. Así, para desarrollar esta idea, podríamos decir que a la hora de determinar si una traducción radical dada es correcta (preparando un manual de traducción), sí hay hechos pertinentes, a saber, los testimonios (conductuales) de los hablantes acerca de la correspondencia pertinente (o latente). Por tanto, sí podría hablarse de traducción correcta: aquella que preserve la conducta discriminativa posible (o más bien que, al no discriminar, la haga inexistente).

El problema es que haber determinado conductualmente cierta correspondencia no supone haber determinado "el significado", pero no porque éste permanezca oculto, sino porque "no hay nada que escrutar", como no había tampoco nada que escrutar en las diversas reducciones conjuntistas de los 
números. Resumiendo: negar hechos pertinentes en la semántica no es negarlos en la traducción, sino sólo a la hora de comparar traducciones. El rationale de Roth es que ciertamente confeccionar un manual de traducción no equivale a haber determinado el significado (no porque sea difícil, sino porque no existe), sino meramente haber escogido un determinado isomorfismo: lo imposible es hallar evidencia relevante para escoger entre isomorfismos.

Esto podría conectarse con el argumento de Dummett según el cual no deberíamos hablar de modelos no estándar como si pudiésemos presuponer la existencia de un modelo estándar con independencia de nuestra manera de construirlo. La aplicación a nuestro problema consistiría en decir que, puesto que no podemos determinar el significado inequívocamente con respecto a un manual de traducción que no haga posibles otras interpretaciones (preservadoras de la conducta para el lenguaje natural, o de las funciones esperadas para las reducciones conjuntistas), entonces tampoco podemos decir que un manual de traducción dado logre determinar el significado "estándar", por mucho que cumpla con todos los requisitos. Volvemos a lo mismo: hay traducciones "correctas", pero no comparación posible entre traducciones distintas igualmente correctas; hay traducciones "verdaderas", pero ninguna determina "el significado verdadero"; hay traducciones "aceptables", pero ninguna es la traducción "estándar".

Termino señalando que, según Putnam, todo este asunto supone un error obvio por parte de Quine, aunque añade que, cuando un gran filósofo comete un error obvio, hay normalmente algo detrás, que en este caso sería la impaciencia quineana ante el hecho de que sus oponentes no asumen la carga de la prueba. Bien, si mis argumentos son correctos entonces es Putnam el equivocado en su interpretación de Quine. Él es también un gran filósofo, así que aventuro la hipótesis de que tras su errónea interpretación de Quine podría haber también cierta impaciencia a la hora de marcar distancias con una postura de la que es realmente difícil diferenciarlo de manera convincente.

\section{Beblogratin}

Benacerraf, P., 1965, "What Numbers Could Not Be", en P. Benacerraf y H. Putnam (comps.) Philosophy of Mathematics, Cambridge Univ. Press, 1983.

Gibson Jr., R.F., 1986, "Translation, Physics and Facts of the Matter", en Hahn y Schilpp, 1986.

Hahn, E.L. y P.A. Schilpp, 1986, The Philosophy of WV. Quine, Open Court, La Salle (IIl.).

Putnam, H., 1962, “The Refutation of Conventionalism”, en Putnam, 1975.

—, 1975, Mind, Language and Reality. Philosophical Papers II, Cambridge Univ. Press, Londres. 
_, 1978, "Realism and Reason", in Meaning and the Moral Sciences, Routledge, Londres.

—, 1980, "Models and Reality", Journal of Symb. Log., no. 45, pp. 464-482, en Putnam, 1983.

- 1981, Reason, Truth and History, Cambridge University Press, Cambridge (Mass.).

- 1983, Realism and Reason, Cambridge University Press, Cambridge.

- 1989, "Model Theory and the 'Factuality of Semantics", en A. George (comp.), Reflections on Chomsky, Basil Blackwell, Oxford, pp. 213-232.

Quine, W.V., 1960, Word and Object, MIT Press, Cambridge (Mass.).

—, 1969, Ontological Relativity and Other Essays, Columbia University Press, Nueva York.

—, 1975, "On Empirically Equivalent Systems of the World", Erkenntnis, no. 9, pp. 313-328.

- 1981, Theories and Things, Harvard Univ. Press.

—, 1986, "Replies", en Hahn y Schilpp, 1986.

Rodríguez-Consuegra, F.A., 1992, "La reducción ontológica y sus problemas", $\mathrm{Cr}$ tica, no. 70, pp. 17-64.

—, 1992a, "Realismo, indeterminación y teoría de modelos", Diálogos, en prensa.

Roth, P.A., 1986, "Semantics without foundations", en Hahn y Schilpp, 1986. 